\title{
¿Es realmente crítico del neoliberalismo el pensamiento de Byung-Chul Han? El problema del presente como crisis sin salida
}

Is the thought of Byung-Chul Han really critical of neoliberalism?

The problem of present as a dead end crisis

Senda Inés Sferco senda.sferco@gmail.com

http://orcid.org/0000-0001-9482-5801

Instituto de Investigaciones Gino Germani; Facultad de Ciencias Sociales;

Universidad de Buenos Aires/ Universidad Nacional del Litoral /

Consejo Nacional de Investigaciones Científicas y Técnicas (Argentina)

\section{Resumen}

Este es un ensayo crítico sobre el pensamiento de Byung-Chul Han. A través de una lectura de sus investigaciones acerca del tiempo presente y respecto de la temporalidad como problema filosófico, este artículo intentará restituir las hipótesis principales del autor buscando dar cuenta 
del tenor de sus implicancias teóricas, metodológicas y políticas. La perspectiva de Han aparece así asentada sobre un binarismo categorial, que, a pesar de su carácter fijante, tiene la capacidad de ir desplazando continuamente sus clasificaciones, a fin de captar todas las posibilidades de inventiva. Nuestra hipótesis de partida es que esta movilidad busca producir una "gramática líquida" que resulta funcional a la lógica de producción de diferencias fomentada en nuestras sociedades neoliberales. El desarrollo del artículo pondrá a prueba este recorrido de lectura, centrándose especialmente en el tratamiento que Han realiza de la noción de "crisis del tiempo" como "disincronía" incapaz de producir una acción bifurcativa. A partir de una recuperación del legado etimológico de "crisis" se develará la inercia de los efectos políticos de esta perspectiva, buscando re posicionar "críticamente" los términos de su debate necesario.

Palabras clave: Temporalidad; Byung-Chul Han; neoliberalismo; crisis; crítica.

\section{Abstract}

This attemps to be a critical essay on the thinking of Byung-Chul Han. Through a comprehensive reading of his research about present time and temporality as a philosophical problem, this article will try to restore the main hypotheses of the author in orderto give an account for the tenor of its theoretical, methodological and political implications. Han's perspective is thus settledon a category binarism, which, despite its fixing nature, has the ability to continually move its classifications, so as to capture all the possibilities of inventiveness. Our starting hypothesis is that this mobility seeks to produce a "liquid grammar" that is functional to the logic of production of differences promoted in our neoliberal societies. The development of the article will test this reading route, especially focusing on Han's treatment of the notion of "time crisis" as "dyssynchrony" incapable of producing a bifurcated action. From a recovery of the etymological legacy of "crisis" the inertia of the political effects of this perspective will be revealed, seeking to "critically" re-locatethe terms of its necessary debate.

Keywords: Temporality; Byung-Chul Han; neoliberalism; crisis; critical.

La perspectiva de Byung-Chul Han no es un mero enfoque de pensamiento más en la miríada de lecturas filosóficas actuales que buscan aprehender los problemas de nuestra contemporaneidad. Es un pensamiento que se plantea un proyecto, que tiene como eje la 
realización de una sistematización filosófica a fin de configurar los términos de una "Segunda Ilustración" (Han, 2016: 94). Esta nominación, que vendría a caracterizar la configuración del tiempo que hoy vivimos, habría heredado las ruinas de la primera llustración, y por eso Han encuentra importante restituir sus basamentos a fin de dar cuenta de su pervivencia en el presente. Su prolífica escritura trata los temas más diversos buscando restituir una configuración de lo actual con la mayor amplitud temática posible: Han escribe acerca del poder, del conocimiento, del inconsciente, del arte y la estética, la multiculturalidad, la temporalidad. Todos estos ámbitos temáticos, aún si diversos e irreductibles entre sí, podrían sin embargo ser abordados a partir de una misma grilla de lectura, ordenada por pares categoriales tales como positivo/negativo, bueno/malo, transparencia/opacidad, etcétera. Estas particiones devienen los pilares de una perspectiva de pensamiento que asienta su legibilidad del mundo en una serie de binarismos ya conformados y siempre extensibles a otros campos de problemas. La cuestión que resulta problemática para el análisis que nos convoca aquí, es que el recurso a la dicotomización conceptual no sólo restituye las encerronas del sujeto antropológico moderno (Foucault, 2003: 331) proponiendo una remanida forma de "representación" de las particiones inclusión/exclusión que funcionan en nuestro mundo, sino que pretende funcionar como recurso "crítico" e innovador en el horizonte de un nuevo pensamiento. En este sentido, aún si el enfoque de Han pueda dar cuenta de cierta pertinencia heurística, sus análisis y articulaciones conceptuales, lamentablemente, rara vez involucran una indagación a nivel de las prácticas o del ámbito de la experiencia, ni vinculación alguna con un registro histórico concreto. Es más, de un modo ciertamente inquietante para cualquier propósito que se quiera "crítico", sus grillas de lectura parecen estar dispuestas únicamente a sostener un nivel teórico que resulta poco fértil a la hora de producir una interpelación efectiva en la lectura "crítica" e "innovadora" del presente que, sin embargo, dice perseguir. En efecto, ¿cómo sería posible dar lugar a una tensión y a una inventiva crítica si todo problema concreto debe necesariamente subsumirse a un binarismo categorial que organice su lectura?

Podríamos pensar que allí radica un nudo conflictivo en el pensamiento de Han que desalienta de cuajo sus propuestas, pero la cuestión es menos prístina y algo más compleja. En efecto, a menudo, cuando examinamos su pensamiento y nos acercamos a dar cuenta de la presencia de un juego teórico especular que impide una producción innovadora y crítica, su escritura se desliza rápidamente desplazando el foco de la mirada, impidiendo una cristalización de sus términos. Esta es, probablemente, la mejor astucia de Han: haber construido una suerte de "gramática líquida" -si nos permitimos evocar las tesis de S. Bauman (2013)-, capaz de expandirse en un plano analítico horizontal, sin espesor ni relaciones de fuerza, sin registro de las singularidades del plano experiencial, pero abarcando sentidos que, por ser 
constantemente desplazados, se muestran en una superficie como gestos críticos respecto de la conformación anterior. Ahora bien, tal como anticipamos y será examinado en este breve estudio, la hipótesis que pondremos a prueba sostiene que dichos desplazamientos no implican una posición "crítica" -al menos según el carácter disruptivo que etimológica y semánticamente comporta este término (Chantraine, 1970: 584)-, sino que más bien están comandando una dinámica de proliferación e incorporación discursiva en bucle que deviene práctica conceptual engullente. Dicho de otro modo, la gramática líquida de Byung Chul Han, lejos de buscar asentar sus términos en una crítica inventiva respecto de lo actual, parece en cambio hallarse a tono con los modos de funcionamiento de las estrategias neoliberales menos explícitas y más eficaces que rigen sobre los modos de producción, creación, pensamientos, acciones y afectos de nuestra actualidad. La "gramática líquida" de Han, en ese sentido, se dispondría a la manera de una red móvil y mutante, a incentivar e identificar diferencias a fin de re-incorporarlas a un sistema que sólo pretende su auto-reproducción. Esta estrategia "nómada" (Deleuze y Guattari, 2004: 390) o, más bien, "parasitaria" (Espósito, 2005: 25) -capaz de hacer de cada rizoma, de cada conjunto relacional, una contaminación que ha de ser fomentada primero, detenida luego, e infinitamente relanzada hacia adentro de una misma lógica-, no se halla sino al servicio de consolidar la necesidad defensiva del paradigma inmunológico que hoy le sive de fundamento. En efecto, si, tomado por un binarismo que organiza la arquitectura de lo posible, occidente es el espacio-tiempo de una lógica que ya está conformada, estas dicotomizaciones no sólo le sirven de basamento, sino que le permiten organizar la constante gestión de producción de diferencias que precisa promover a nivel social y cultural. Han nos brinda varias descripciones de ello: es necesario hoy más que nunca performarse identitariamente, volverse empresarios de sí mismos, capacitarse, asumir más diversidad, esas son las encerronas sin salida de nuestro mundo. Ahora bien, para que efectivamente no haya salida, es necesario que el nivel teórico excluya el nivel práctico, que lo subsuma a una operación que se convalide siempre desde sí misma, insertando sus conceptualizaciones en un plano de legibilidad inmunológica que no precisa hacer recurso a ningún trazo de experiencia que lo confronte y lo obligue a reposicionar críticamente las bases de su legibilidad. Si el imperativo de esta producción diferencial es funcional a la vitalidad neoliberal de nuestros sistemas de gobierno, también será funcional, como veremos, a la anticipación permanente de toda producción de novedad.

En este texto, entonces, se intentará dar cuenta de las implicancias teóricas, metodológicas y políticas de esta perspectiva, a partir del tratamiento de uno de los tópicos en los que probablemente sea más difícil intentar aprehender estos corrimientos: nos referimos al abordaje que Byung-Chul Han hace de la cuestión de la crisis del tiempo. En efecto, si la temporalidad es la dimensión deviniente y evanescente por excelencia para el pensamiento 
filosófico, este campo de problematización -a nuestro entender- ofrecerá al autor una posibilidad de tematización, o, mejor dicho, de "representación" de las oscilaciones con las que busca discurrir sus construcciones categoriales cuando se enfrentan a sus limitaciones fijantes. En consecuencia, nos preguntamos si Byung-Chul Han no construye una "gramática líquida" que resulta de algún modo implícitamente eficaz a la estrategia de incorporación discursiva con la que se nutre el régimen neoliberal, esta eficacia sita en el carácter escurridizo de su escritura podría ser explicitada a partir de su tematización de la temporalidad.

Cabría reconocer, con todo, la eficaz potencialidad descriptiva de la gramática del tiempo presente que la perspectiva de Byung-Chul Han repone. En efecto, su aparato conceptual ofrece una "imagen" de nuestro tiempo, que, emulando el gesto heideggeriano y blumenbergiano respecto de nuestra imagen del mundo (cf. 1996, 2007), busca elaborar una representación del campo de problemas de nuestra actualidad. Tal como nos advierte a lo largo La sociedad de la transparencia (2013), Psicopolítica (2014), Topología de la violencia ([2013] 2016) y La expulsión de lo distinto (2017) nuestra sociedad contemporánea estaría marcada por una experiencia caída (cf. Benjamin, 1998), transida por la pérdida de opacidad y de diferencia de las subjetividades que la componen. Esta mundialidad, compelida por una exigencia de transparencia propia de los modos de consumo de nuestras sociedades neoliberales, se hallaría condenada a la iteración de lo mismo como espacio-tiempo positivo. En esta configuración, dice Byung-Chul Han, el tiempo hace "crisis" y resulta impotente para organizar una acción que pueda convalidarse en términos histórico-emancipatorios. De esta constatación efectiva surge el hilo rojo que, a partir de una caracterización de las "crisis del tiempo", va dando lugar a las argumentaciones del autor que nos ocuparán a continuación.

\section{Las “crisis del tiempo” de Byung-Chul Han}

En su libro El aroma del tiempo. Un ensayo filosófico sobre el arte de demorarse (2009), el autor se aboca especialmente a la problematización conceptual de las temporalidades que rigen la experiencia y la producción subjetiva en nuestra actualidad, comenzando por identificar algunos matices que -a pesar de que la liquidez de su enfoque no los desarrolla-, a nuestro entender, marcan algunas pistas que pueden ser productivas para inteligir las complejidades del tiempo presente.

Byung-Chul Han parte de la afirmación de que hoy "...el tiempo carece de un ritmo ordenador. De ahí que pierda el compás". Esta dispersión resulta de la constatación de que "...no hay nada que rija el tiempo" (2009: 6). En consecuencia, sin parámetro rector, “...la vida ya no se 
enmarca en una estructura ordenada ni se guía por las coordenadas que generen una duración" (2009: 7). El tiempo, así, "da tumbos (...) sin rumbo alguno", y ésta es, al decir de Byung-Chul, la crisis de disincronía que sufre nuestro mundo actual.

Su argumentación se asienta en la positividad del diagnóstico posmoderno, para el cual, desde "el final de la historia ninguna duración guía al tiempo" (2009: 41). Asumiendo dicho "final de la historia" como condición de posibilidad de nuestro presente, el paradigma de consumo de la era de la información (cf. Baudrillard, 1991; Bauman, 2013) habría hecho, a su vez, de la "atomización" del tiempo la única vivencia posible. Así, tal como nos explica el autor, "...los acontecimientos se desprenden con rapidez los unos de los otros, sin dejar una marca profunda, sin llegar a convertirse en una experiencia" (Han, 2009: 57). Puesto que su temporalidad se halla vaciada de negatividad y de opacidad (Han, 2013) el tiempo, transparente, sólo convalidaría la instancia efímera de su carácter surgiente, impotente a la hora de generar marca o espesor con fuerza de diferencia. Así, aplanado en una superficie, según el autor, no habría caso de intentar registrar en él relieves experienciales ni subjetivos, puesto que ya no tiene "duración".

En efecto, para Byung-Chul Han, y de un modo ciertamente reductivo, la duración habría muerto junto con las narrativas de la aceleración -de corte histórico y teleológico-, propias de la Modernidad.

\begin{abstract}
El tiempo se precipita como una avalancha porque ya no cuenta con ningún sostén en su interior. Cada punto del presente, entre los cuales ya no existe ninguna fuerza de atracción temporal, hace que el tiempo se desboque, que los procesos se aceleren sin dirección alguna, y precisamente por no tener dirección alguna no se puede hablar de aceleración. La aceleración, en sentido estricto, presupone caminos unidireccionales (Han, 2009: 18).
\end{abstract}

A diferencia de esa época unidireccionalizada, nuestra modernidad tardía "post-histórica" no estaría atravesada por la problemática de la aceleración (que para el autor devino una mera percepción subjetiva) (2009: 7), sino por una "dispersión temporal" multidireccional que no permite experimentar ningún tipo de duración (2009: 8). En consecuencia, a diferencia del tiempo mítico e histórico, que buscaba colmar los vacíos y los intervalos de la temporalidad mediante sus narrativas explicativas, el tiempo atomizado característico de nuestra actualidad, acortaría al máximo la dilación entre los sucesos, anulando cualquier posibilidad de duración y de vinculación entre sus términos. De esta manera, en la lógica de iterabilidad de nuestro mundo actual "no hay nada que ligue los acontecimientos entre ellos generando una relación, es decir, una duración" (Han, 2009: 44). 
Estas afirmaciones, con las que ciertamente se apresura demasiado la concatenación de los problemas que su retórica líquida va considerando, acarrean consecuencias políticas muy serias. Frente a ellas, no podemos no preguntarnos... ¿Qué estrategia argumentativa sostiene la efectuación de un paralelo entre "crisis de duración" a nivel discursivo y "crisis de relación" a nivel de la experiencia? ¿Cuál es el modo de veridicción de "la crisis" de nuestro mundo actual que configura esta superposición teórico-práctica? ¿No hay en dicho desplazamiento sintagmático - de la duración a la relación- una visibilidad reductiva, al servicio de asumir que hoy ya no podemos contar con ningún tipo de vínculo durable, es decir, que ninguna relación está en grado de probarse efectivamente subjetivante y ser capaz de reposicionar los términos de la crisis de nuestra experiencia?

$\mathrm{Si}$, tal como advierte el autor, hoy "...la vida ya no se enmarca en una estructura ordenada ni se guía por unas coordenadas que generan una duración" (Han, 2009: 7), y la "crisis de disincronía" es la clave para inteligir las complejidades de esta temporalidad díscola, ByungChul Han podría tener acceso a la dispersión temporal propia de la experiencia atomizada del tiempo de nuestra actualidad haciendo del eco del lamento hamletiano -time is out of joints- un son contemporáneo. Y, munido del carácter activo que diagnostica su "crisis" (Chantraine, 1970), convertir a la disincronía en una llave conceptual capaz de descomponer en un espectro de matices experienciales y subjetivos una experiencia que, ciertamente, ya no puede ser cifrada como unívoca.

Sin embargo, de acuerdo a sus argumentos, la crisis de la experiencia actual no implica únicamente una crisis de las gramáticas de la duración, sino que resulta asimismo identificable como crisis de relación. No tendríamos entonces tampoco aquí, en sentido estricto, una "disincronía" (puesto que en una lógica de la identidad como dinámica de adecuación sujeto/objeto no hay discordancia entre sus términos), sino una mera superposición de planos que el autor va uniendo en bucle a costa de ir diluyendo sus diferentes especificidades. Ya nos ocuparemos seguidamente de este problema -que podríamos simpáticamente llamar, en honor a su proliferación alegórica, el "barroquismo" de Byung-Chul Han-; tomemos nota, por ahora, del carácter escurridizo de la "gramática líquida" con el que caracterizamos su elaboración teórica, que aquí desplaza, reduce y diluye en una misma igualación "experiencia", "duración" y "relación", anulando el potencial crítico -histórico, particular y contingente- de la crisis que, en cambio, su diagnóstico disincrónico señala. En efecto, si la resultante de su lectura en lugar de abrir una mirada sobre lo disyunto busca reunir tres crisis en una, y todas pueden ser leídas desde el mismo prisma categorial, ya no hay necesidad de atender a su especificidad, ni a las relaciones de fuerza o a la heterogeneidad de un plano empírico que permita chequear $\mathrm{y} / \mathrm{o}$ hacer advenir la urgencia de una elaboración conceptual a nivel teórico. No hay en Byung-Chul 
Han, de hecho, a lo largo de su obra, ningún interés de acceso a esta materialidad del orden de las prácticas, ni a las modalidades subjetivas de una singularidad experiencial. En todo caso, como anticipamos, la impotencia crítica de su mirada es útil para situar en el final de la historia una lectura del tiempo presente afín a las necesidades de los mecanismos reproductores de las discursividades neoliberales engullentes de toda incitada diferencialidad. En este sentido, si el diagnóstico de un vacío a nivel experiencial y subjetivo deja un tiempo cuya capacidad actuante ya no puede convalidarse en términos de duración histórica ni de lazo social, el problema principal, en consecuencia, radicará en la pregunta acerca de qué tipo de acción se probará capaz de desandar críticamente estas crisis. Dicho de otro modo, si junto a él pensamos que la "crisis de disincronía" es la clave heurística para inteligir la temporalización subjetiva de nuestro presente, ¿qué acción deviene entonces efectivamente capaz de desandar dicha crisis?; ¿hay en Byung-Chul Han un deseo emancipatorio? ¿Cómo podríamos concebir la situacionalidad de esta acción, si se anticipa incapaz de hacer experiencia?

\section{Una acción a los tumbos}

Han indica que este tiempo sin ritmo ordenador no sólo da cuenta de una atomización experiencial y subjetiva, sino que emerge, asimismo, como síntoma de una acción que anda a los tumbos, asumiendo haber perdido "el rumbo", otrora brindado por las narrativas de la historia como paradigma filosófico de ordenamiento temporal. Sin sostén que opere de punto de gravitación y de espesor (Han, 2009: 55), hoy ya no sería posible trazar las coordenadas de esta acción ni desde la temporalidad direccionada por la necesidad de telos en una filosofía de la historia, ni haciendo pie en la negatividad de un andar a contrapelo de la historia que permita bifurcar el continuum de la herencia catastrófica de la Modernidad (Benjamin, 1940). Esto es así, advierte, porque en la lógica positiva de la transparencia de nuestra actualidad, "nada es decisivo; nada hace corte" (Han, 2009: 57).

Sin embargo, y a fin de cotejar la arqueología analítica que él mismo propone, recordemos que "crisis", por mandato etimológico, pertenece justamente a la semántica de cortes y decisiones bifurcativas comandada por su radical *krr (Chantraine, 1970: 584). Esta filiación, que por otra parte comparte con la idea de "crítica", impone la exigencia de efectuar una acción decisiva que permita una apertura en el medio empírico, un parte aguas respecto de las lógicas hasta el momento convalidadas para su reproducción. Por lo tanto, es preciso identificar y temporalizar las condiciones que puedan preparar su advenimiento: registrar la particularidad del conflicto de la coyuntura en crisis que reclama activamente su emergencia, asumir un posicionamiento 
particularmente situado capaz de problematizar histórica, experiencial y subjetivamente las relaciones disincrónicas entre sus términos, calibrar los matices de las prácticas que habrán de sostener sus consecuencias, inferir los diferentes modos posibles, en fin, de acción factible. En efecto, no es sino en ese "aquí y ahora" de las condiciones de posibilidad que sostendrán su corte, donde la crisis viene a marcar un diagnóstico "crítico" en el marco del cual -tal como nos enseñaba la medicina hipocrática-, deviene urgente actuar. Ahora bien, si bien para ByungChul Han éste es un problema que ilumina las dificultades de nuestra modernidad tardía, su perspectiva analítica no parece poder aprehenderlo. De hecho, inculpa a la pérdida de sostén por la duración a la parestesia de una acción que en nuestro tiempo habría quedado detenida, incapaz de producir irrupción subjetiva alguna. Ahora bien, junto con el historiador conceptual Reinhart Koselleck (2007) -por citar un ejemplo entre varias perspectivas efectivamente críticas-, podríamos recordar a Byung-Chul Han que las voces que han dado en decir históricamente la crisis, aún en la Modernidad, nos muestran que su acción nunca tuvo asegurada la duración. Al contrario, al igual que el médico hipocrático, el corte crítico de la crisis ha debido actuar siempre en una episteme falible e incompleta, en medio de causas nunca totalmente anticipables, en pleno riesgo pharmakon. Asumiendo ese agon irreconciliable de la acción crítica, que, como nos advierte Koselleck (2007), hereda la patogénesis del imaginario social y político moderno, la crisis produce una apertura que ha de rearmar lazos a fin de sostener y reelaborar la experiencia que su novedad impone. ¿Qué busca bifurcar, entonces, la crisis de disincronía de Byung-Chul Han?

Ciertamente, el filósofo coreano no es el primero en hacer de la disyunción la ocasión de una crisis, y aun si la idea de "crisis de disincronía" (Han, 2009: 6) presentada (aunque lamentablemente no elaborada) por el autor, se muestra a primera luz capaz de una heurística interesante, diagnostica un problema que en verdad no resulta problemático desde la perspectiva de Byung-Chul Han. En efecto, de un modo muy revelador, la imagen del mundo que comporta la enunciación "crisis de disincronía" podría reforzar una apuesta crítica de alta intensidad, puesto que la crisis aparece identificada doblemente: tanto por el descalzamiento que expresa su caracterización de la experiencia como crisis disincrónica de temporalidades siempre disyuntas, como por la crítica a los modos de legilibidad convalidados para leerla que impone "la crisis" como sujeto de predicación. En otras palabras, si convenimos que cuando hablamos de disincronía temporal referimos al menos a dos temporalidades cuyo carácter disyunto da cuenta del espacio-tiempo crítico de una terceridad, hablar de disincronía es ya hablar de crisis, por lo tanto, referir a su crisis vendría a redoblar la necesidad de una creación conceptual dispuesta a dar legibilidad al plano de lo que efectivamente buscaría nombrar. Pero el autor no desglosa esta deriva. Tampoco echa luz en las conceptualizaciones que ya dieron 
en vincular las crisis del tiempo a una caída de la experiencia heredada de la modernidad (Benjamin, 1998), o que buscaron situar en el registro de las crisis las condiciones para una articulación posible entre pasado y futuro capaz de reposicionar lo actual en un horizonte de expectativas diferentes (Kosselleck, 1993; Hartog, 2003). Byung-Chul Han, tímidamente, busca alumbrar un problema que luego no indaga, al menos a nivel de las tensiones que invita a problematizar la puesta en uso de la noción de "crisis", y en consecuencia su producción categorial resulta meramente ilustrativa.

Incluso, de un modo más simple, si en nuestra sociedad de la trasparencia el tiempo atomizado ya no produciría experiencia, no habría locus descalzado ni tempus de disincronía que analizar. La conceptualización de temporalidades disímiles y heterogéneas -que se desprende de un modo ciertamente interesante de un abordaje del modo de acontecer disincrónico del tiempono tendría asidero. Aún si Byung-Chul Han advierte que "...la vida humana se empobrece cuando se queda sin cualquier intermedio. La cultura humana también es rica en intermedios" (2009: 82), habiendo perdido su capacidad de proliferación de lazos, esta noción sólo fluctúa entre oximorones teóricos y líquidos que ya no precisan ser movilizados hacia un nivel empírico. Ni siquiera a nivel teórico resulta una oportunidad conceptual dispuesta a dar legibilidad a diversos matices de apertura y de diferencialidad con el objetivo de actualizar una episteme del presente que hoy precisa más que nunca la visión de sus relieves y su opacidad. Lamentablemente, Byung-Chul Han polemiza un problema que luego no aborda, y, una vez más, rápidamente, su enfoque se ocupa de completar líquidamente su elástico andamiaje categorial binario, rellenando la fisura que, sin embargo, había contribuido a señalar.

El dato que resulta aún más grave debido a la "ingenuidad" de sus consecuencias políticas, es que en este libro Byung-Chul Han se propone brindar pistas propositivas, en clave emancipatoria, señalando una salida urgente y plausible capaz de rescatar a la temporalidad de nuestra experiencia del flagelo de la disincronía y la parestesia de la tardo modernidad. Tal como podemos sospechar a esta altura del análisis, dicha recuperación del tiempo no posicionará la salida de su crisis en la acción crítica, sino en la acción pasiva -o mejor dicho, la “inacción"-, propia de la práctica de la contemplación. 


\section{La temporalidad propositiva de Byung-Chul Han}

Desde las primeras páginas de El aroma del tiempo (2009), de un modo ciertamente peculiar ya que no lo efectúa en todos sus libros, Byung-Chul Han presenta las bases programáticas para desandar la crisis de duración de nuestro presente. Según sus propias palabras:

No se trata de lamentar la pérdida de la época de la narración. El final de la narración, el final de la historia, no tiene por qué traer consigo un vacío temporal. Al contrario, da lugar a la posibilidad de una vida que no necesita la teología ni la teleología y que, a pesar de ello, tiene su propio aroma. Pero requiere una revitalización de la vita contemplativa (Han, 2009: 9).

Byung-Chul Han revisita la concepción aristotélica de animal laborans -con una breve referencia a la interpretación hegeliana y arendtiana de esta cuestión-, en función de dar cuenta de la enajenación productiva del mundo actual y de la importancia de dar lugar y tiempo a una vida contemplativa. Frente a la hiperkinesia de la aceleración de nuestras sociedades, el autor llama a "perder el tiempo" y a revalorizar los elementos duraderos de la vida humana. La pauta emancipatoria como práctica de resistencia frente a la cultura laborans es la "demora". El ocio agustiniano, el antecedente. La contemplación aristotélica, el horizonte. Al decir de ByungChul Han, "...la crisis temporal solo se superará en el momento en que la vita activa, en plena crisis, acoja de nuevo la vita contemplativa en su seno" (2009: 9). Una vez más, la estrategia restitutiva del autor atiende al uso de su contrario, puesto que frente a una experiencia atomizada y sin aroma:

\footnotetext{
El tiempo comienza a tener aroma cuando adquiere una duración, cuando cobra una tensión narrativa o una tensión profunda, cuando gana en profundidad y amplitud, en espacio. El tiempo pierde el aroma cuando se despoja de cualquier estructura de sentido, de profundidad, cuando se atomiza o se aplana, se enflaquece o se acorta. (...) El tiempo se precipita, se agolpa para equilibrar una falta de Ser esencial, aunque no lo consigue, porque la aceleración por sí misma no proporciona ningún sostén. Solo hace que la falta de ser resulte incluso más penetrante. (Han, 2009: 45).
}

Evidentemente, esta apuesta por la redensificación de la falta del Ser esencial, se asienta en la línea de la estrategia de Heidegger en Ser y tiempo (2003), quien insistía en que estamos arrojados a un mundo que no hemos elegido, cuya aperturidad siempre inquietante se nos revela en una estructura temporal sin fundamento histórico o teleológico que nos reclama dicha 
tarea temporalizante. El hombre, el ser-en-el-mundo, ha de sostener, entonces, ahí la preocupación de dar cuenta, una y otra vez, de su existencia del modo más auténtico posible, produciendo un ejercicio de interpelación que requiere siempre de una incitación recíproca entre un registro óntico y ontológico de la existencia. Ahora bien, si es verdad que para Heidegger la autenticidad ha de ser conquistada, y allí radica una tarea angustiosa y vital que ha de tomar cierta distancia de los mandatos de la mundaneidad, el hecho de la existencia siempre está condicionado fácticamente y nunca puede agotar su permanente inconclusión (Heidegger, 2003: 41-45). De hecho, la autenticidad de la inquietud por el desocultamiento, más que en la mera contemplación, ha de activarse en la apertura capaz de movilizar un cuidado (Sorge) puesto que esta es la ocupación (Besorgen) como modo de estar-en-elmundo.

Sin embargo, la altura contemplativa que prefiere Byung-Chul Han no ancla la pregunta por la existencia en una relación de cuidado. Más bien busca aislarse del mundo, abstraerse de su opacidad, salvarse de la fetidez enviciada de sus complicaciones. Desde allí, tiene dispensada la acción que conllevaría la inquietud de cuidado de los otros, o de un sí mismo -en los términos heideggereanos o incluso foucaultianos de un cuidado de sí mismos y de los otros (1984)- siempre reposicionado en la incitación a amplificar un lazo subjetivante como materialidad relacional y crítica de la vida en común.

Recordemos, para terminar, que la crisis de disincronía de nuestro presente para Byung-Chul Han se conceptualiza como crisis de duración y crisis de relación. Sobre el final del libro, el autor suma a ellas, una "crisis de identidad": "La atomización de la vida supone una atomización de la identidad" (2009: 7). No tendría caso seguir indagando a qué figura reductiva (puesto que se halla atomizada), correspondería la identidad que añora el autor, porque ya no nos queda mucho misterio: ciertamente se trata de un "hombre" -puesto que no hay tampoco "mujer" ni diferencialidad o pluralidad de géneros cualesquiera en sus proposiciones aparentemente diversas. Probablemente sea el hombre que, según su argumento, veridiccionaba el rumbo de la acción "certera" y la duración como aceleración uni-progresista de un Humanismo que, como imaginario-centro de Europa, aún no puede organizar su pesimismo (Benjamin, 1988). Tal vez la impotencia crítica de la crisis de Byung-Chul Han esté a tono con cierto síntoma de envejecimiento filosófico, que todavía habla en nombre de un occidente que no asume la necesidad de hacer otros montajes con los restos de sus caídas, ni puede complicar sus universales (Cassin, 2016) por la vía de una experiencia efectivamente disincrónica.

Como instancia de salida a la crisis, la "contemplación" representa, a la vez que un tiempo de algún modo suspendido, una topografía que se despliega en un espacio-tiempo 'más allá' de 
toda experiencia. Mirando desde arriba, sobrevolando sin tocar nuestra experiencia ya caída, el andamiaje conceptual de Byung-Chul Han inscribe la tarea de la filosofía en la búsqueda de depuración de un aroma que, tal vez lo salve de la complejidad empírica, pero en todo caso ciertamente le impide abordar analíticamente el síntoma que identifica: la crisis de disincronía tiene en el postulado contemplativo su primera disyunción (o la primera complicidad con el régimen que critica) puesto que, sin analizar la heterogeneidad temporal de las relaciones efectivas que componen las tensiones que hacen crisis a nivel de la experiencia, su salida se exime de la tarea de interrogar cómo podría producir una acción capaz de interrumpir lo dado y rearmar lazos que se sostengan emancipatorios. Podríamos sospechar, en consecuencia, que el aroma del tiempo de Byung-Chul Han no puede con los olores de una historia que precisa ser retemporalizada. Su gramática líquida refuerza, en cambio, una soledad melancólica puesto que alude a una nostalgia de sensaciones e ideas, a una duración y un rumbo certero de una acción que en verdad la Modernidad nunca efectivamente tuvo. Desde este punto sin materialidad, lejos de reposicionar los términos para su desenajenación efectiva, el animal laborans consolida definitivamente la positividad de su inclinación a la servidumbre voluntaria.

\section{Notas}

(1) La llamamos "líquida", puesto que la operación argumentativa de Chul Han se inscribe en la impronta epistemológica denunciada por Zygmunt Bauman (2013), quien advertía que nuestro tiempo postindustrial correspondía a la imagen de una "modernidad líquida". Según esta idea, los modelos sólidos de institucionalidad y socialidad propios de la modernidad, se habrían flexibilizado, desregulado y llenados de incertidumbre. La liquidez es el modo rector, entonces, que para Bauman viene a nombrar un modo de experiencia y una producción de subjetividad contemporánea que ya no tiene en la relación forma-contenido una identidad capaz de objetivarla, sino cuya labilidad sin materialidad ni compromiso toma la forma del recipiente cualquiera sea que pueda contenerla.

(2) Recordemos que pharmakon es un vocablo griego que permite semantizar las ambivalencias ligadas a las consecuencias de una acción. En efecto, refiere a las pócimas, que, en el saber médico, son tanto capaces de curar y restablecer el equilibrio del enfermo como de envenenarlo fatalmente.

\section{Bibliografía}

Baudrillard, J. (1991). La transparencia del mal. Ensayo sobre los fenómenos extremos. Barcelona: Anagrama.

Bauman, Z. (2013). La cultura en el mundo de la modernidad líquida. Fondo de Cultura Económica: Buenos Aires.

Question, Vol. 1, N. 0 61, enero-marzo 2019. ISSN 1669-6581

Instituto de Investigaciones en Comunicación | Facultad de Periodismo y Comunicación Social | Universidad Nacional de La Plata La 
Benjamin, W. (2008). Tesis sobre la historia y otros fragmentos. Madrid: Itaca.

Benjamin, W. (1998). Iluminaciones IV. Madrid: Taurus.

Blumenberg, H. (2007). Tiempo de la vida y tiempo del mundo. Valencia: PreTextos.

Cassin, B. (2016). Éloge de la traduction. Compliquer l'universel. Paris: Fayard.

Chantraine, P. (1970). Dictionnaire étymologie de la langue grecque. Histoire des mots (Tomo II). E-K. Paris: Klincksieck.

Deleuze, G. y Guattari, F. (2004). Mil Mesetas. Capitalismo y Esquizofrenia (J. Vásquez Pérez y U. Larraceleta (Traductores). Valencia: PreTextos.

Han, B-C. (2009). El aroma del tiempo. Un ensayo filosófico sobre el arte de demorarse (Traducción de Paula Kuffer). Barcelona: Herder.

Han, B-C. (2013). La sociedad de la transparencia. Barcelona: Herder.

Han, B-C. (2014). Psicopolítica. Barcelona: Herder.

Han, B-C. (2016). Topología de la violencia. Barcelona: Herder.

Han, B-C. (2017). La expulsión de lo distinto. Barcelona: Herder.

Espósito, R. (2005). Inmunitas: protección y negación de la vida. Barcelona: Amorrortu.

Foucault, M. (2003). Las palabras y las cosas. Una arqueología de las Ciencias Humanas. Siglo XXI: Buenos Aires.

Foucault, M. (2008). Le gouvernement de soi et des autres. Cours au Collège de France. 19821983. Paris: Seuil.

Heidegger, M. (2003). Ser y Tiempo. Madrid: Trotta.

Heidegger, M. (1996). Caminos de bosque. Madrid: Alianza.

Hartog, F. (2003). Régimes d'historicité. Présentisme et expérience du temps. Paris: Du Seuil.

Koselleck, R. (1993). Futuro pasado. Para una semántica de los tiempos históricos. Barcelona: Paidós.

Koselleck, R. (2001). Los estratos del tiempo. Barcelona: Paidós.

Koselleck, R. (2007). Crítica y Crisis. Un estudio sobre la patogénesis del mundo burgués. Madrid: Trotta.

Koselleck, R. y Richter, M. W. (2006). Crisis. Journal of the History of Ideas. Vol. 67, No. 2, 357400.

La Boétie, E. (1992). Discurso sobre la servidumbre voluntaria. Buenos Aires: Colihue. 